\title{
Vedolizumab trough level monitoring in inflammatory bowel disease: a state-of-the- art overview
}

\author{
Lieven Pouillon $^{1 *}$, Séverine Vermeire ${ }^{2}$ and Peter Bossuyt ${ }^{1}$
}

\begin{abstract}
Background: Therapeutic drug monitoring involves therapeutic modifications based on the measurement of drug levels and antidrug antibodies. The viability of therapeutic drug monitoring in vedolizumab-treated patients with inflammatory bowel disease remains questioned.

Main body: Accumulating evidence from clinical trials and real-world data suggests that an exposure-efficacy relationship may exist for vedolizumab in inflammatory bowel disease, but results are not as straightforward as they are for anti-tumour necrosis factor-a therapy. Robust target vedolizumab trough levels are currently missing, since available data are heterogenous and prospective, interventional pharmacokinetic-pharmacodynamic studies are lacking. The positioning of vedolizumab drug monitoring in therapeutic algorithms is yet to be defined.

Conclusion: Therapeutic drug monitoring has the potential to improve the outcome parameters of vedolizumabtreated patients with inflammatory bowel disease. Before the therapeutic drug monitoring of vedolizumab can be implemented in a widespread fashion, prospective studies are needed to evaluate the effect of vedolizumab dose optimisation. These studies should focus on objective disease markers and vedolizumab drug levels, and define thresholds for optimal drug exposure.
\end{abstract}

Keywords: Crohn's disease, Inflammatory bowel disease, Therapeutic drug monitoring, Vedolizumab, Ulcerative colitis

\section{Background}

Monoclonal antibodies directed against tumour necrosis factor- $\alpha$ (anti-TNF) have conventionally been the cornerstone of the treatment of moderate-to-severe inflammatory bowel disease (IBD) [1]. Nevertheless, approximately one-third of biological-naïve patients experience a primary non-response to anti-TNF therapy, and secondary loss of response is seen in almost half of the patients over time [2, 3]. In the search to enlarge the therapeutic armamentarium, vedolizumab has been shown to be effective in both Crohn's disease (CD) and ulcerative colitis (UC) [4, 5]. Although the mechanism of action of vedolizumab is not fully understood, it is presumed to act primarily by binding to $\alpha 4 \beta 7$ integrin, which is predominantly expressed by a subset of gastrointestinal-homing T-lymphocytes. Vedolizumab

\footnotetext{
* Correspondence: lievenpouillon@icloud.com

${ }^{1}$ Imelda Gl Clinical Research Centre, Imeldaziekenhuis Bonheiden, Bonheiden, Belgium

Full list of author information is available at the end of the article
}

thereby prevents the interaction of $\alpha 4 \beta 7$ integrin with mucosal addressin cell adhesion molecule-1 (MAdCAM-1) on the surface of mucosal endothelial cells, and inhibits the migration of T-lymphocytes into the bowel tissue [6].

Therapeutic drug monitoring involves measuring drug levels and antidrug antibodies with adjustment of the dose when needed, and is particularly of interest because drug exposure - rather than the administered dose - is related to response to biologicals. Since anti-TNF therapy uses weight-based dosing, theoretically more flexibility is possible compared with vedolizumab therapy, in which a fixed dose is administered. In anti-TNF-treated patients, therapeutic drug monitoring has been shown to be a valuable tool to guide decision-making in patients with insufficient response to therapy [7, 8]. Although registered trials in $\mathrm{CD}$ and $\mathrm{UC}$ have suggested an exposure-efficacy relationship with vedolizumab $[4,5,9,10]$, the viability of therapeutic drug monitoring for vedolizumab remains less clear. To improve insights, several

(c) The Author(s). 2019 Open Access This article is distributed under the terms of the Creative Commons Attribution 4.0 International License (http://creativecommons.org/licenses/by/4.0/), which permits unrestricted use, distribution, and reproduction in any medium, provided you give appropriate credit to the original author(s) and the source, provide a link to the Creative Commons license, and indicate if changes were made. The Creative Commons Public Domain Dedication waiver (http://creativecommons.org/publicdomain/zero/1.0/) applies to the data made available in this article, unless otherwise stated. 
researchers have recently studied vedolizumab trough levels and clinical, endoscopic and histological outcome parameters in IBD patients [11-17].

The aims of this mini-review are to give an overview of the currently available knowledge and future perspectives of therapeutic drug monitoring in vedolizumab-treated patients.

\section{Search strategy}

We searched for relevant manuscripts in PubMed/MEDLINE, EMBASE (Excerpta Medica Database) and Cochrane CENTRAL, from their inception until March 5th, 2019. The following keywords were included, alone or in combination: "Crohn's disease", "ulcerative colitis", "inflammatory bowel disease", "vedolizumab", "trough levels", "serum levels", and "therapeutic drug monitoring". Relevant articles published in English were critically reviewed. Bibliographies of included articles were searched, and experts in IBD were consulted to identify additional studies. Only full papers were considered for review; data exclusively available in abstract format were not taken into consideration.

\section{Exposure-efficacy relationship}

An exposure-efficacy relationship for vedolizumab has been suggested in clinical trials and most real-world cohorts (Table 1).

\section{Clinical trials}

Post-hoc analyses of the GEMINI trials were the first to reveal that higher vedolizumab trough levels during induction therapy correlated with higher clinical remission rates in IBD [9]. Interestingly, induction trough levels of less than $17 \mu \mathrm{g} / \mathrm{mL}$ for $\mathrm{UC}$, and less than $16 \mu \mathrm{g} / \mathrm{mL}$ for $\mathrm{CD}$, were associated with clinical remission rates similar to that of placebo. The exposure-efficacy relationship was steeper for UC than for CD [9]. The same authors recently published a propensity score-based case-matched analysis of UC patients in the GEMINI trials, aiming to characterise the relationship between vedolizumab exposure and response using patient-level data, and adjusting for confounding factors affecting vedolizumab drug clearance and serum levels [10]. Potential target vedolizumab trough levels were $37.1 \mu \mathrm{g} / \mathrm{mL}$ at week 6 during induction, $18.4 \mu \mathrm{g} / \mathrm{mL}$ at week 14 after induction, and $12.7 \mu \mathrm{g} / \mathrm{mL}$ during maintenance treatment [10].

\section{Real-world cohorts}

Data from the two largest available real-world cohorts to date confirmed a link between higher vedolizumab exposure and achieving better outcomes [13, 15]. In a retrospective Belgian study, vedolizumab trough levels > $30 \mu \mathrm{g} / \mathrm{mL}$ at week $2,>24 \mu \mathrm{g} / \mathrm{mL}$ at week 6 , and $>14 \mu \mathrm{g} /$ $\mathrm{mL}$ during maintenance therapy, correlated with higher clinical and endoscopic effectiveness endpoints in IBD patients [13]. Endoscopic remission was achieved in significantly more patients with UC than patients with CD, even though a diagnosis of UC was not an independent predictor of higher vedolizumab trough levels [13]. In a cross-sectional study from the USA, patients in steroid-free clinical and biologic remission had significantly higher vedolizumab trough levels than those who did not, although differences between both groups were small [15].

Multicentric data from France showed that vedolizumab trough levels below $18.5 \mu \mathrm{g} / \mathrm{mL}$ at week 6 were associated with the need for additional doses during the first 6 months of therapy [11]. A similar cut-off proved to be the only independent variable associated with mucosal healing within the first year of vedolizumab treatment [14]. In the latter study, only median vedolizumab trough levels at week 6 , and not at week 2 or week 14 , differed between patients with and without mucosal healing within the first year after treatment initiation [14]. A similarly designed study from another group also noted higher vedolizumab trough levels during induction in patients with steroid-free endoscopic remission after one year of treatment; however, only trough levels at week 2 differed significantly [17]. Interestingly, higher vedolizumab trough levels during maintenance therapy for UC have also been associated with histological remission, a distinct treatment target linked with better clinical outcomes [16]. Nevertheless, only a small number of patients were included, and confirmation in larger, independent cohorts remains necessary [16]. In conflict with the majority of data, one study found no exposure-efficacy relationship for vedolizumab [12].

\section{Pharmacokinetics/pharmacodynamics}

Vedolizumab has a primarly linear clearance at therapeutic concentrations, with no difference between patients with $\mathrm{CD}$ and UC [18, 19]. Pooled population data from the GEMINI program identified low albumin concentration and very high body mass to be predictors of accelerated vedolizumab clearance [18]. Indeed, most real-world studies seem to confirm the clinical importance of these factors, linking them with lower drug levels and worse therapeutic outcomes $[12,13]$. Low albumin also influences infliximab and adalimumab clearance rates, and is in part a surrogate marker of disease severity [20]. In this regard, more severe disease at initiation of vedolizumab therapy, reflected in low baseline albumin, but also in low haemoglobin and/or high C-reactive protein (CRP) levels, has been associated with lower vedolizumab drug levels during treatment and a lower likelihood of achieving therapeutic targets $[9,13,17$, 21]. There is an inverse relationship between vedolizumab drug levels and CRP [12, 14, 21, 22]. However, whether lower drug levels exacerbate disease activity, or whether 


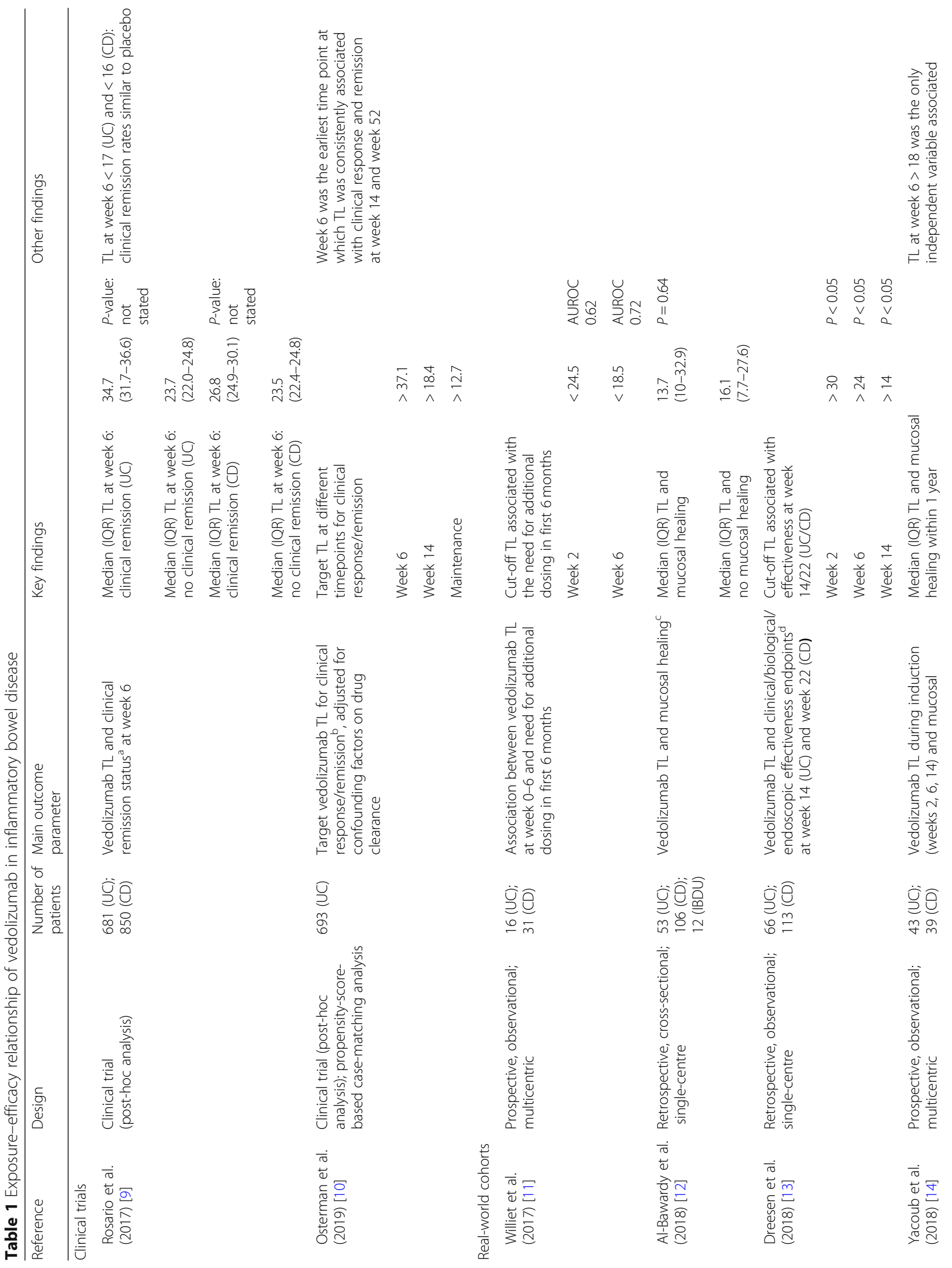




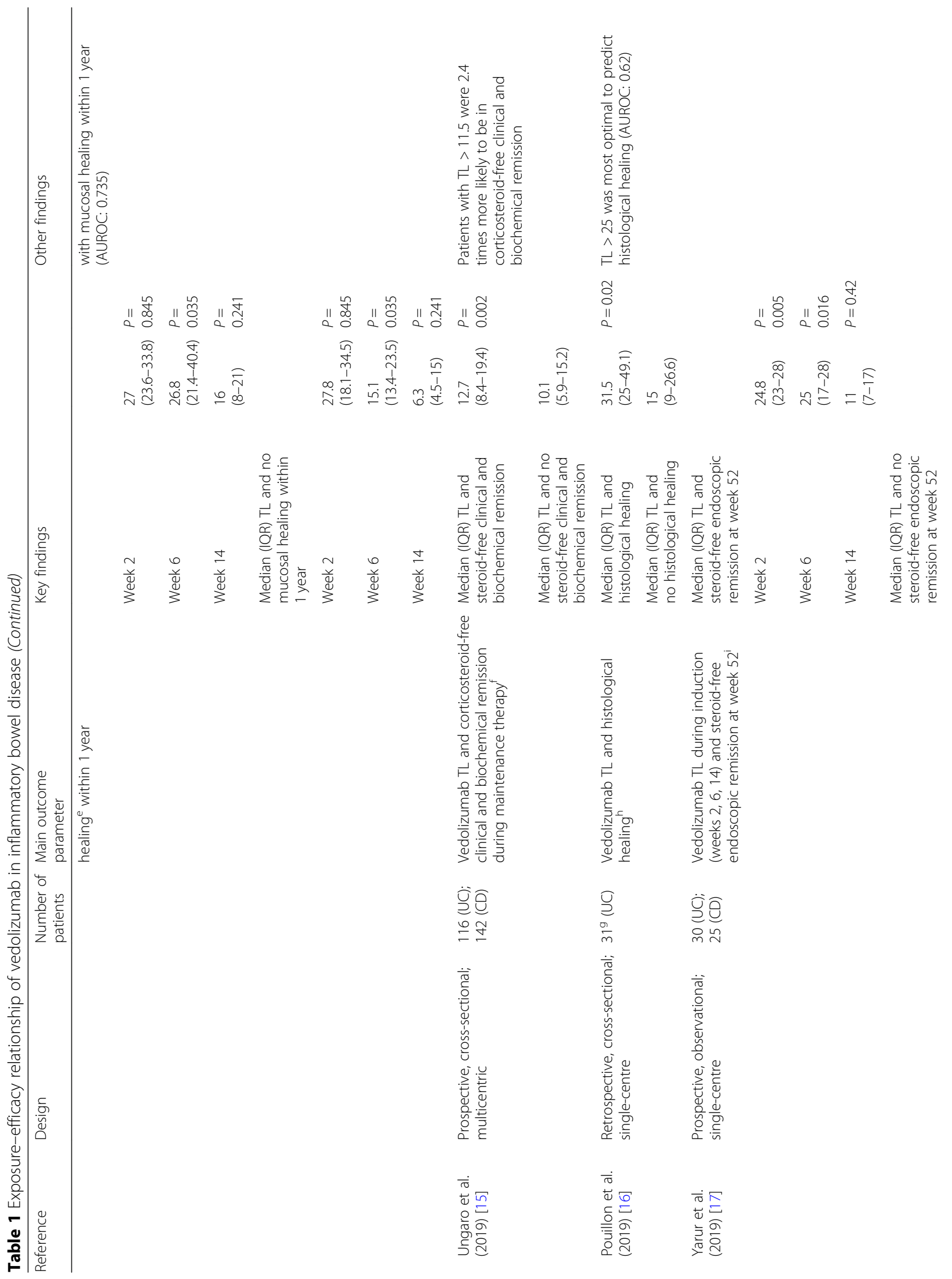




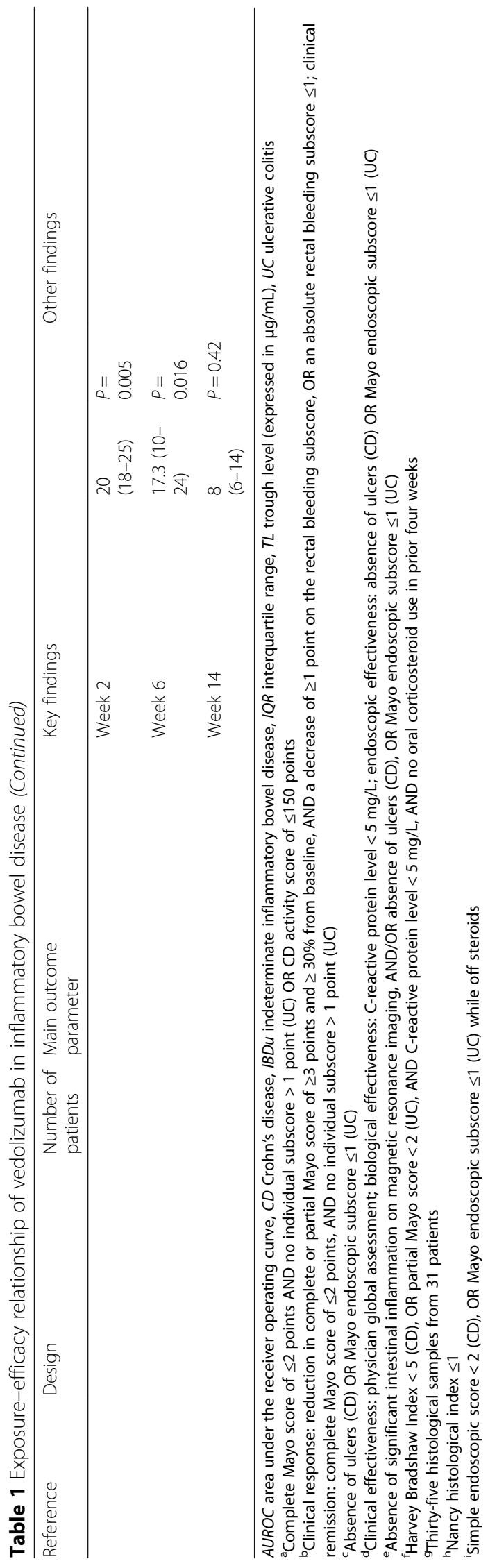


higher disease activity results in lower drug levels, remains uncertain.

\section{Immunogenicity}

Immunogenicity of vedolizumab in randomised controlled trials was low, with less than $5 \%$ of patients having at least one sample testing positive for anti-vedolizumab antibodies at any time, and fewer than $1 \%$ of patients with persistently positive anti-vedolizumab antibodies $[4,5]$. This has been confirmed in all available real-world data so far, even when using a drug-resistant assay [23, 24]. It might explain why adding an immunomodulator to vedolizumab therapy seems neither to enhance drug levels nor to restore therapeutic response $[14,25]$.

\section{Promising features and potential drawbacks}

Despite the growing body of evidence for an exposureefficacy relationship for vedolizumab, data remain difficult to interpret and do not allow firm recommendations to be drawn for clinical practice (Fig. 1). Most real-world studies were not designed prospectively with the primary aim of making inferences about the drug exposure-response profile. Heterogeneous study designs, including different outcome parameters and definitions, make direct comparisons difficult. Further more, all but one [10] study analysed data on a population level instead of an individual level, refraining to adjust for confounding factors that influence vedolizumab drug clearance and serum levels. Lastly, in the absence of comparative data between different assays used to measure vedolizumab drug levels, it cannot be ruled out that absolute differences in cut-offs across studies are secondary to disagreement of the utilised assays.

Therapeutic drug monitoring of TNF antagonists has shown to improve response and remission rates in IBD patients $[26,27]$. It proved to aid mostly in determining the therapeutic strategy in anti-TNF-treated patients who are losing response [28], and led to major cost savings [29]. Extrapolation of these data to vedolizumab-treated patients is not yet possible because of the lack of robust evidence. Furthermore, the utility of therapeutic drug monitoring for anti-TNF has been

\section{STRENGTHS}

- Growing body of evidence for

an exposure-efficacy

relationship for vedolizumab

- Increased knowledge about

factors influencing vedolizumab

drug clearance and serum levels

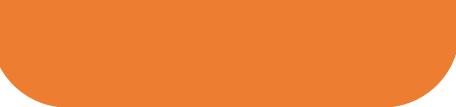

\section{OPPORTUNITIES}

- Improve outcome parameters of vedolizumab-treated IBD

patients

- Determine patients that would

benefit from vedolizumab dose

optimization

\section{WEAKNESSES}

- Heterogeneous data do not yet

allow robust target vedolizumab

trough levels to be defined

- Prospective, interventional

PK/PD studies are currently

lacking

\section{THREATS}

- Low immunogenicity of

vedolizumab might limit clinical

utility of TDM

- Positioning of TDM in

therapeutic algorithms remains

unclear

Fig. 1 SWOT analysis (strengths, weaknesses, opportunities and threats) summarizing the promising features and potential drawbacks of vedolizumab trough level monitoring in inflammatory bowel disease (IBD). PK/PD: pharmacokinetic/pharmacodynamic; TDM: therapeutic drug monitoring 
strengthened by the likelihood of antidrug antibody development, since this should prompt clinicians to initiate a different TNF antagonist rather than to switch to another class of biological. Currently, no other anti-integrin is widely available, and antibody development against vedolizumab is a rare event, so the benefit of therapeutic drug monitoring for vedolizumab may be more marginal, and its positioning in therapeutic algorithms is yet to be defined.

\section{Future directions}

The mechanisms underlying non-response or loss of response to vedolizumab are unrevealed. Almost complete occupancy of integrin $\alpha 4 \beta 7$ is seen on peripheral and intestinal T-cells from vedolizumab-treated IBD patients, regardless of serum levels [22]. Together with low immunogenicity, vedolizumab probably has additional modes of action. In this respect, vedolizumab treatment was recently shown to exert substantial effects on macrophage populations and expression of molecules involved in microbial sensing, chemoattraction and regulation of the innate effector response, suggesting at least a role for the innate immune system in the therapeutic efficacy of vedolizumab [30].

Dose optimisation of vedolizumab restores responsiveness in half of patients experiencing a secondary loss of response [31]. In a small, retrospective analysis of IBD patients who underwent vedolizumab dose optimisation, mean change from the baseline of vedolizumab trough levels at month 3 after dose optimisation was numerically higher in the group of responders versus the group of non-responders [32]. Before reactive therapeutic drug monitoring in vedolizumab-treated patients with insufficient response can be widely recommended, prospective studies must explore the effect of dose optimisation on objective disease markers and changes in vedolizumab drug levels. In this regard, the results of a continuing vedolizumab dose optimisation randomized controlled trial, ENTERPRET (NCT03029143), are eagarly awaited. This type of study should also allow further clarification of desired trough level intervals, both during induction and maintenance treatment.

Conflicting data mean the proactive use of therapeutic drug monitoring in symptom-free patients is heavily debated for anti-TNF [27, 33]. It remains to be seen whether cost-effectiveness for vedolizumab will ever be shown.

\section{Conclusion}

Data from randomised registration trials and subsequent real-world cohorts suggest an exposure-efficacy relationship of vedolizumab in patients with $\mathrm{CD}$ and $\mathrm{UC}$, but important drawbacks for therapeutic drug monitoring of vedolizumab still exist. Before therapeutic drug monitoring for vedolizumab can be widely recommended, prospective studies must evaluate the effect of vedolizumab dose optimization. These studies should focus on objective disease markers and vedolizumab drug levels, and define thresholds for optimal drug exposure.

\section{Acknowledgements \\ Not applicable. \\ Funding \\ Not applicable.}

\section{Availability of data and materials}

Not applicable.

\section{Authors' contributions}

LP drafted the manuscript, with contributions from SV and PB. All authors read and approved the final version of the manuscript.

\section{Authors' information}

SV is a senior clinical investigator of the Research Foundation - Flanders (FWO).

Ethics approval and consent to participate Not applicable.

\section{Consent for publication}

Not applicable.

\section{Competing interests}

LP received travel grants from Abvvie, Ferring and Takeda. SV has served as a speaker for AbbVie, MSD, Pfizer, Takeda, Hospira and Ferring; received consultant fees from AbbVie, MSD, Pfizer, Takeda, Hospira, Ferring, Mundipharma, Second Genome, Galapagos, Genentech/Roche, Janssen, Celgene; served as an advisory board member for AbbVie, MSD, Pfizer, Takeda, Hospira, Ferring, Mundipharma, Second Genome, Galapagos, Genentech/Roche, Janssen, Celgene; and received research grants from MSD, AbbVie, Pfizer, Takeda. PB received educational grants from AbbVie, Mundipharma, Pfizer and Janssen; speaker fees from AbbVie, Takeda and Pfizer;and advisory board fees from Hospira, Janssen, MSD, Mundipharma, Roche, Pfizer, Takeda, Sandoz and Pentax.

\section{Publisher's Note}

Springer Nature remains neutral with regard to jurisdictional claims in published maps and institutional affiliations.

\section{Author details}

${ }^{1}$ Imelda Gl Clinical Research Centre, Imeldaziekenhuis Bonheiden, Bonheiden, Belgium. ${ }^{2}$ Department of Gastroenterology and Hepatology, University Hospitals Leuven, KU Leuven, Leuven, Belgium.

Received: 7 January 2019 Accepted: 11 April 2019

Published online: 08 May 2019

\section{References}

1. Pouillon L, Bossuyt P, Peyrin-Biroulet L. Considerations, challenges and future of anti-TNF therapy in treating inflammatory bowel disease. Expert Opin Biol Ther. 2016;16:1277-90.

2. Singh S, Fumery M, Sandborn WJ, Murad MH. Systematic review and network meta-analysis: first- and second-line biologic therapies for moderate-severe Crohn's disease. Aliment Pharmacol Ther. 2018;48:394-409.

3. Singh S, Fumery M, Sandborn WJ, Murad MH. Systematic review with network meta-analysis: first- and second-line pharmacotherapy for moderate-severe ulcerative colitis. Aliment Pharmacol Ther. 2018;47:162-75.

4. Sandborn WJ, Feagan BG, Rutgeerts P, Hanauer S, Colombel JF, Sands BE, et al. Vedolizumab as induction and maintenance therapy for Crohn's disease. N Engl J Med. 2013;369:711-21. 
5. Feagan BG, Rutgeerts P, Sands BE, Hanauer S, Colombel JF, Sandborn WJ, et al. Vedolizumab as induction and maintenance therapy for ulcerative colitis. N Engl J Med. 2013;369:699-710.

6. Wyant T, Fedyk E, Abhyankar B. An overview of the mechanism of action of the monoclonal antibody vedolizumab. J Crohns Colitis. 2016;10:1437-44.

7. Mitrev N, Vande Casteele N, Seow CH, Andrews JM, Connor SJ, Moore GT, et al. Review article: consensus statements on therapeutic drug monitoring of anti-tumour necrosis factor therapy in inflammatory bowel diseases. Aliment Pharmacol Ther. 2017:46:1037-53.

8. Vande Casteele N, Herfarth H, Katz J, Falck-Ytter Y, Singh S. American Gastroenterological Association Institute technical review on the role of therapeutic drug monitoring in the management of inflammatory bowel diseases. Gastroenterology. 2017;153:835-857.e6.

9. Rosario M, French JL, Dirks NL, Sankoh S, Parikh A, Yang H, et al. Exposureefficacy relationships for vedolizumab induction therapy in patients with ulcerative colitis or Crohn's disease. J Crohns Colitis. 2017;11:921-9.

10. Osterman MT, Rosario M, Lasch K, Barocas M, Wilbur JD, Dirks NL, et al. Vedolizumab exposure levels and clinical outcomes in ulcerative colitis: determining the potential for dose optimisation. Aliment Pharmacol Ther. 2019;49:408-18.

11. Williet N, Boschetti G, Fovet M, Di Bernado T, Claudez P, Del Tedesco E, et al. Association between low trough levels of vedolizumab during induction therapy for inflammatory bowel diseases and need for additional doses within 6 months. Clin Gastroenterol Hepatol. 2017;15:1750-1757.e3.

12. Al-Bawardy B, Ramos GP, Willrich MAV, Jenkins SM, Park SH, Aniwan S, et al. Vedolizumab drug level correlation with clinical remission, biomarker normalization, and mucosal healing in inflammatory bowel disease. Inflamm Bowel Dis. 2019;25:580-6.

13. Dreesen E, Verstockt B, Bian S, de Bruyn M, Compernolle G, Tops S, et al. Evidence to support monitoring of vedolizumab trough concentrations in patients with inflammatory bowel diseases. Clin Gastroenterol Hepatol. 2018;16:1937-1946.e8.

14. Yacoub W, Williet N, Pouillon L, Di-Bernado T, De Carvalho Bittencourt M, Nancey $S$, et al. Early vedolizumab trough levels predict mucosal healing in inflammatory bowel disease: a multicentre prospective observational study. Aliment Pharmacol Ther. 2018;47:906-12.

15. Ungaro R, Yarur A, Jossen J, et al. Higher trough vedolizumab concentrations during maintenance therapy are associated with corticosteroid-free remission in inflammatory bowel disease. J Crohns Colitis. 2019. https://doi.org/10.1093/ecco-jcc/jjz041.

16. Pouillon L, Rousseau H, Busby-Venner H, De Carvalho Bittencourt M, Choukour M, Gauchotte G, et al. Vedolizumab trough levels and histological healing during maintenance therapy in ulcerative colitis. J Crohns Colitis. 2019. https://doi.org/10.1093/ecco-jcc/jjz029.

17. Yarur AJ, Bruss A, Naik S, Beniwal-Patel P, Fox C, Jain A, et al. Vedolizumab concentrations are associated with long-term endoscopic remission in patients with inflammatory bowel diseases. Dig Dis Sci. 2019. https://doi. org/10.1007/s10620-019-05570-1.

18. Rosario M, Dirks NL, Gastonguay MR, Fasanmade AA, Wyant T, Parikh A, et al. Population pharmacokinetics-pharmacodynamics of vedolizumab in patients with ulcerative colitis and Crohn's disease. Aliment Pharmacol Ther. 2015:42:188-202.

19. Rosario M, Dirks NL, Milch C, Parikh A, Bargfrede M, Wyant T, et al. A review of the clinical pharmacokinetics, pharmacodynamics, and immunogenicity of vedolizumab. Clin Pharmacokinet. 2017;56:1287-301.

20. Hemperly A, Vande Casteele N. Clinical pharmacokinetics and pharmacodynamics of infliximab in the treatment of inflammatory bowel disease. Clin Pharmacokinet. 2018:57:929-42.

21. Schulze H, Esters P, Hartmann F, Stein J, Christ C, Zorn M, et al. A prospective cohort study to assess the relevance of vedolizumab drug level monitoring in IBD patients. Scand J Gastroenterol. 2018;53:670-6.

22. Ungar B, Kopylov U, Yavzori M, Fudim E, Picard O, Lahat A, et al. Association of vedolizumab level, anti-drug antibodies, and a4 $\beta 7$ occupancy with response in patients with inflammatory bowel diseases. Clin Gastroenterol Hepatol. 2018;16:697-705.e7.

23. Bian S, Dreesen E, Tang HT, Compernolle G, Peeters M, Van Assche G, et al. Antibodies toward vedolizumab appear from the first infusion onward and disappear over time. Inflamm Bowel Dis. 2017;23:2202-8.

24. Van den Berghe N, Verstockt B, Tops S, Ferrante M, Vermeire S, Gils A Immunogenicity is not the driving force of treatment failure in vedolizumab-treated inflammatory bowel disease patients. J Gastroenterol Hepatol. 2018. https://doi.org/10.1111/jgh.14584.
25. Gouynou C, Peyrin-Biroulet L. Letter: addition of methotrexate neither restores clinical response nor improves the pharmacokinetic profile of vedolizumab-treated patients. Aliment Pharmacol Ther. 2017:46:1019-20.

26. Steenholdt C, Brynskov J, Thomsen $\bigcirc \varnothing$, Munck LK, Fallingborg J, Christensen LA, et al. Individualised therapy is more cost-effective than dose intensification in patients with Crohn's disease who lose response to antiTNF treatment: a randomised, controlled trial. Gut. 2014;63:919-27.

27. Vande Casteele N, Ferrante M, Van Assche G, Ballet V, Compernolle G, Van Steen $\mathrm{K}$, et al. Trough concentrations of infliximab guide dosing for patients with inflammatory bowel disease. Gastroenterol. 2015;148:1320-9 e3.

28. Afif W, Loftus EV, Faubion WA, Kane SV, Bruining DH, Hanson KA, et al. Clinical utility of measuring infliximab and human anti-chimeric antibody concentrations in patients with inflammatory bowel disease. Am J Gastroenterol. 2010;105:1133-9.

29. Martelli L, Olivera P, Roblin X, Attar A, Peyrin-Biroulet L. Cost-effectiveness of drug monitoring of anti-TNF therapy in inflammatory bowel disease and rheumatoid arthritis: a systematic review. J Gastroenterol. 2017;52:19-25.

30. Zeissig S, Rosati E, Dowds CM, Aden K, Bethge J, Schulte B, et al. Vedolizumab is associated with changes in innate rather than adaptive immunity in patients with inflammatory bowel disease. Gut. 2019;68:25-39.

31. Peyrin-Biroulet L, Danese S, Argollo M, Pouillon L, Peppas S, GonzalezLorenzo $\mathrm{M}$, et al. Loss of response to vedolizumab and ability of dose intensification to restore response in patients with Crohn's disease or ulcerative colitis: a systematic review and meta-analysis. Clin Gastroenterol Hepatol. 2019;17:838-46.e2.

32. Gouynou C, Pouillon L, Rousseau H, Zallot C, Baumann C, Peyrin-Biroulet L. Early changes in the pharmacokinetic profile of vedolizumab-treated patients with IBD may predict response after dose optimisation. Gut. 2019; 68:178-9.

33. D'Haens G, Vermeire S, Lambrecht G, Baert F, Bossuyt P, Pariente B, et al. Increasing infliximab dose based on symptoms, biomarkers, and serum drug concentrations does not increase clinical, endoscopic, and corticosteroidfree remission in patients with active luminal Crohn's disease. Gastroenterol. 2018;154:1343-51 e1.

\section{Ready to submit your research? Choose BMC and benefit from:}

- fast, convenient online submission

- thorough peer review by experienced researchers in your field

- rapid publication on acceptance

- support for research data, including large and complex data types

- gold Open Access which fosters wider collaboration and increased citations

- maximum visibility for your research: over $100 \mathrm{M}$ website views per year

At BMC, research is always in progress.

Learn more biomedcentral.com/submissions 